Muséologies

Les cahiers d'études supérieures

muséologies

\title{
Mise en perspective chiasmique des histoires de l'art global au Canada
}

\author{
Alice Ming Wai Jim
}

Volume 9, numéro 1, 2018

Monde des Arts / Arts des mondes

URI : https://id.erudit.org/iderudit/1052630ar

DOI : https://doi.org/10.7202/1052630ar

Aller au sommaire du numéro

\section{Éditeur(s)}

Association Québécoise de Promotion des Recherches Étudiantes en Muséologie (AQPREM)

\section{ISSN}

1718-5181 (imprimé)

1929-7815 (numérique)

Découvrir la revue

\section{Citer cet article}

Jim, A. M. W. (2018). Mise en perspective chiasmique des histoires de l'art global au Canada. Muséologies, 9(1), 97-111. https://doi.org/10.7202/1052630ar

\section{Résumé de l'article}

This article offers a critical perspective on the pedagogical direction of what I call "global art histories" in Canada by addressing the apparent impasse posed by the notion of what is euphemistically called "ethnocultural art" in this country. It examines different interpretations of the latter chiefly through a survey of course titles from art history programs in Canada and a course on the subject that I teach at Concordia University in Montreal. Generally speaking, the term "ethnocultural art" refers to what is more commonly understood as "ethnic minority arts" in the ostensibly more derisive discourses on Canadian multiculturalism and cultural diversity. The addition of the term "culture" emphasizes the voluntary self-definition involved in ethnic identification and makes the distinction with "racial minorities." "Ethnocultural communities," along with the moniker "cultural communities" (or "culturally diverse" communities), however, is still often understood to refer to immigrants (whether recent or long-standing), members of racialized minorities, and even First Nations, Inuit, and Métis peoples. Not surprisingly, courses on ethnocultural art histories tend to concentrate on the cultural production of visible minorities or ethnocultural groups. However, I also see teaching the subject as an opportunity to shift the classification of art according to particular geographic areas to consider a myriad of issues in myriad of issues in the visual field predicated on local senses of belonging shaped by migration histories and "first" contacts. As such, ethnocultural art histories call attention to, but not exclusively, the art of various diasporic becomings inexorably bound to histories of settler colonialism and Indigenous sovereignty. This leads me to reflect on some aspects of Quebec's internal dynamics concerning nationalism and ethnocultural diversity that have affected the course of ethnocultural art histories in the province. I argue that the Eurocentric hegemonic hold of ethno-nationalist discourses on art and art history can be seen with particular clarity in this context. Moreover, I suggest that these discourses have hindered not only the awareness and study of art by so-called culturally diverse communities but also efforts to offer a more global, transnational, and heterogeneous (or chiastic) sense of the histories from which this art emerges. In today's political climate, the project that is art history, now more than ever, needs to address and engage with the reverse parallelism that chiastic perspectives on the historiography of contemporary art entail. My critique is forcefully speculative and meant to bring together different critical vocabularies in the consideration of implications of the global and ethnic turns in art and art history for the understanding of the other. I engage in an aspect less covered in the literature on the global turn in contemporary art, namely the ways in which the mutual and dialectical relation between "cultural identity," better described as a "localized sense of belonging" (Appadurai) and the contingency of place may shape, resist, or undermine the introduction of world or global art historical approaches in specific national institutional sites. I argue a more attentive politics of engagement is required within this pedagogical rapprochement to address how histories not only of so-called non-Western art but also diasporic and Indigenous art are transferred holistically as knowledge, if the objective is to shift understandings of the other by emphasizing points of practice in art history as a field, rather than simply the cultural productions themselves. I propose the term "global art histories" as a provisional rubric that slants the study of globalism in art history to more explicitly include these kinds of located intercultural negotiations.
Tous droits réservés (C) Association Québécoise de Promotion des Recherches Étudiantes en Muséologie (AQPREM), 2018
Ce document est protégé par la loi sur le droit d'auteur. L'utilisation des services d'Érudit (y compris la reproduction) est assujettie à sa politique d'utilisation que vous pouvez consulter en ligne.

https://apropos.erudit.org/fr/usagers/politique-dutilisation/ 
Article cinq

Mise en perspective chiasmique des histoires de l'art global au Canada

Alice Ming Wai Jim 


\section{Alice Ming Wai Jim est titulaire de la Chaire de recherche de l'Université Concordia sur les histoires de l'art ethnoculturel et professeure au département d'histoire de l'art de l'Université Concordia à Montréal.}

Cet article est une version remaniée d'un texte publié originellement sous le titre "Dealing with Chiastic Perspectives: Global Art Histories in Canada." dans Lynda Jessup, Keri Cronin, et Kirsty Robertson (dir.), Negotiations in a Vacant Lot: Studying the Visual in Canada, Montréal et Kingston, McGill-Queen's University Press, 2014, p. 66-90. 


\section{Introduction}

Dans le présent article, je propose une réflexion préliminaire sur l'orientation pédagogique que l'on donne maintenant, au Canada, à une discipline que je me permets d'appeler " histoires de l'art global ", ou Global art histories en anglais. Parallèlement, j'aborde l'impasse que semble créer la notion d'" art ethnoculturel " - pour reprendre un euphémisme prisé dans notre pays. Par ailleurs, je me penche sur certains aspects des dynamiques intrinsèques du nationalisme et de la diversité ethnoculturelle au Québec. Je m'intéresse plus particulièrement à leur influence sur le cours des histoires de l'art ethnoculturel dans cette province. Je soutiens que la portée eurocentrique, hégémonique, du discours ethnonationaliste en matière d'art et d'histoire de l'art transparaît manifestement dans les contextes étudiés. En outre, j'avance que ce discours nuit non seulement à la sensibilisation à l'expression créatrice et à l'étude de l'art dans de soi-disant communautés culturellement diversifiées, mais également aux efforts déployés pour attribuer un sens plus mondialiste, transnational et hétérogène (ou chiasmique) aux histoires dont émerge cet art.

Théoriques d'entrée de jeu, mes observations visent à agencer différents vocabulaires critiques dans l'examen des répercussions des tournants ethnique et mondialiste, en art et en histoire de l'art, sur la compréhension de l'autre. Elles ne se préoccupent guère des possibilités et des limites propres à un éventuel rapprochement entre l'histoire de l'art mondial, d'une part, et ces nouveaux domaines que sont les études de l'art mondial et l'histoire de l'art global, d'autre part. Rappelons au passage que cette dernière discipline a pour objet l'art qui se fait depuis une vingtaine d'années ${ }^{1}$. Du tournant global qu'a récemment amorcé l'art contemporain ont vite découlé de nombreux travaux d'érudition, surtout dans les milieux américains et européens. Ces textes ciblent plus précisément le "mondialisme ", pour reprendre le mot de l'historien de l'art James Elkins, qui teinte la notion de contemporanéité. Ils s'attardent notamment aux modalités et aux perspectives pour l'histoire de l'art d'envergure globale que provoquent migrations, diasporas et capitalismes à l'échelle du monde ${ }^{2}$.

Dans ce texte, j'approfondis une question peu documentée. En effet, je traite des diverses formes que revêt l'interrelation dialectique entre « identité culturelle »- ou, pour employer une expression plus exacte, sentiment d'appartenance circonscrit ${ }^{3}$ - et " contingence du lieu " dans le façonnement, la consolidation ou la fragilisation des approches de l'histoire de l'art en matière d'art global ou mondial, à l'intérieur de cadres institutionnels nationaux précis. Je soutiens qu'un tel rapprochement
1 Le présent article reprend la distinction que fait Hans Belting entre " art mondial " et " art global " (respectivement world art et global art en anglais). " Expression synonyme du patrimoine artistique des autres [...] à l'échelle universelle [...], l'art mondial englobe la plupart des cultures extraoccidentales dont l'héritage artistique est préservé par des musées de type "empire colonial”. „ Pour sa part, l'art global « est systématiquement conçu dans une intention artistique; sur ce plan, il se rapproche de l'art contemporain. " Source: BELTING Hans, "Contemporary Art as Global Art ", The Global Art World, 2009, p. 44 (traduction libre). Surtout, il convient de bien discerner l'« histoire de l'art mondial " des " études de l'art mondial " (respectivement world art history et World Art Studies en anglais). Expression créée par John Onians en 1992, "études de l'art mondial " désigne une approche artistique qui émane d'une perspective global transcendant la chronologie et la géographie, et qui fait appel à tous les points de vue imaginables, mais néanmoins pertinents sur le plan disciplinaire: culture visuelle, études culturelles, anthropologie, neuroscience, philosophie, etc. Source: ONIANS John, World Art Studies, 2008, p. 206-209 (traduction libre). En plein essor, ce « nouveau " champ d'études - ainsi que ses corollaires, notamment l'" histoire de l'art global " et les " histoires comparatives de l'art " - soustend plusieurs obligations: appréhender la réalité de plus en plus globale et interdisciplinaire de l'art et du discours connexe; reconnaître le caractère non seulement inadéquat mais indéfendable de l'interprétation de l'art extraoccidental au moyen de méthodes occidentales; et remettre en question l'approche eurocentrique traditionnelle des canons de l'histoire de l'art occidental forgés à l'ère du colonialisme.

2 ELKINS James, "Can We Invent a World Art Studies?", World Art Studies, 2008, p. 107. Par ailleurs, les ouvrages Is Art History Global?, publié sous la direction de James Elkins (2007), et The Global Art World, publié sous la direction de Hans Belting et d'Andrea Buddensieg (2009), traitent d'études préliminaires effectuées en littérature.

3 APPADURAI Arjun, "The Production of Locality", Counterworks: Managing the Diversity of Knowledge, 1995, p. 204-225. 
pédagogique exige une politique d'engagement plus diligente, car il doit expliquer comment les histoires - j'insiste sur le pluriel - portant non seulement sur l'art dit " extraoccidental ", mais aussi sur l'art diasporique et l'art autochtone, sont véhiculées selon le paradigme holistique du transfert des connaissances. Il s'agit ici de modifier la compréhension de l'autre en insistant sur des éléments saillants de la pratique de l'histoire de l'art en tant que discipline plutôt que de considérer simplement des productions culturelles concrètes. Par conséquent, je propose comme titre provisoire " histoires de l'art global ». À mon avis, le terme excède les frontières de l'étude du mondialisme en histoire de l'art. Mieux, il englobe explicitement différents types de négociations interculturelles localisées.

\section{L'amorce d'un programme de recherche}

Selon la compréhension que j'en ai, les histoires de l'art ethnoculturel tentent de revoir la classification des arts, de la moduler en fonction de zones géographiques données, afin de prendre en considération une myriade de questions qui touchent le domaine du visuel et découlent de sentiments d'appartenance locaux façonnés par des expériences migratoires et des contacts « initiaux ». C'est ainsi que les histoires de l'art ethnoculturel attirent l'attention - sans toutefois la monopoliser - sur la production artistique d'une variété de diasporas en devenir. J'essaie personnellement d'éviter de tomber dans le piège, mais les cours universitaires portant sur ce sujet ont souvent tendance à se limiter à l'étude des artefacts culturels de minorités visibles ou de groupes ethnoculturels. Dans le discours - ostensiblement ironique - sur le multiculturalisme canadien et la diversité culturelle, l'expression " art ethnoculturel » renvoie généralement au concept plus répandu d'" œuvres produites par des membres des minorités ethniques "-nommément à l'art, considéré comme inférieur, que produisent ces " éternels immigrants, [ces] étrangers domestiques ${ }^{4}$ ", que sont les Canadiens d'ascendance non européenne. La référence à la « culture » fait ressortir l'autodéfinition délibérée que sous-tend le principe d'appartenance ethnique et établit une distinction avec l'expression " minorité raciale". Cependant, le sens du terme " communauté ethnoculturelle ", ainsi que les synonymes " communauté culturelle " et " communauté "culturellement diversifiée" ", est encore bien souvent associé à l'idée d'immigrant (peu importe qu'il soit récent ou bien établi) ou de membre des minorités racialisées, voire des Premières Nations ou des collectivités inuites ou métisses.

Au Canada, depuis 1981 et notamment à la suite de l'adoption de la Charte des droits et libertés, l'expression " minorité raciale " a cédé la place à celle de " minorité visible ». Selon la Loi sur l'équité en matière d'emploi, promulguée en 1995 par le gouvernement canadien, font partie des minorités visibles « les personnes, autres que les Autochtones [c'est-àdire Amérindiens, Métis ou Inuits], qui ne sont pas de race blanche ou qui n'ont pas la peau blanche ". Bien que cette formulation réponde aux objectifs énoncés en matière de lutte contre les inégalités sociales fondées sur l'appartenance raciale, il ne fait aucun doute qu'elle reflète le système de croyances propre au suprémacisme blanc que sous-entendait le terme employé précédemment. Par ailleurs, aux fins du recensement canadien, l'expression " origine ethnique " désigne le ou les groupes ethniques ou culturels dont faisaient partie les ancêtres du répondant; l'origine ethnique ne doit pas être confondue avec la citoyenneté ou la nationalité. Compte tenu de l'émergence des déclarations d'ascendance ethnique nationale, ce dernier point peut toutefois prêter à discussion. Dans le recensement de 1996, le gentilé «Canadien " servait d'exemple pour illustrer la notion de groupe ethnique. Lors du même exercice, la mention " Québécois » - qui figurait jusque-là sous la rubrique "Origines françaises " (c'est-à-dire Français ou Acadien) - s'est retrouvée dans la catégorie "Autres origines ". 
Au Québec, où je donne des cours d'histoire de l'art ethnoculturel et d'histoire de l'art global, $60,2 \%$ des participants au recensement ont indiqué - exclusivement ou notamment "Canadien" au titre de leur origine ethnique. De même, 28,9\% ont fait état d'origines françaises. Enfin, 1,9\% des répondants ont signalé des origines québécoises; un tiers d'entre eux, soit près de 50000 personnes, ont mentionné d'autres origines ${ }^{5}$. Au fil des ans, la question sur l'origine ethnique a été reformulée maintes et maintes fois. Aussi, de recensement en recensement, les comparaisons relatives à l'ampleur de la diversité ethnique, à l'échelle nationale ou autre, se sont avérées problématiques. C'est tout particulièrement le cas au Québec. Comme l'explique le sociologue Victor Piché,

de plus en plus, la catégorisation ethnique cède place à la catégorisation linguistique. D'une part, les statistiques ethniques ne sont plus exploitables; d'autre part, la situation reflète les politiques québécoises en matière de langue et d'immigration, lesquelles définissent l'intégration comme un élément des critères linguistiques et non pas ethniques ${ }^{6}$.

En 1977, la mise en œuvre par le Parti québécois - parti politique indépendantiste de centre gauche - de la Charte de la langue française ( loi $101 »)$, qui faisait du français l'unique langue officielle du Québec, a réussi à renforcer le statut de cette langue ainsi qu'à généraliser son utilisation dans la province. Conséquence inattendue de la Charte, le multilinguisme s'est largement répandu au Québec, et une nouvelle diversité ethnique est apparue au sein de la majorité sociologique constituée par les Franco-Québécois et les groupes multiethniques d'expression française.
En ce qui a trait aux minorités visibles, la publication en 2005 d'une étude prévisionnelle de Statistique Canada sur la diversité ethnoculturelle de la population canadienne a fait les manchettes d'un océan à l'autre. En effet, " environ un Canadien sur cinq, soit entre $19 \%$ et $23 \%$ de la population du pays, pourrait appartenir à un groupe de minorités visibles d'ici 2017, au moment où le Canada célébrera son $150^{\mathrm{e}}$ anniversaire $"^{7}$. D'après l'étude, le nombre et la proportion de l'ensemble des membres des minorités visibles devaient doubler avant 2017. Dans le Grand Toronto (la ville la plus multiculturelle au Canada) et à Vancouver, ces derniers devaient former plus de la moitié de la population. Résultat: les deux agglomérations devaient comporter, pour la toute première fois, une " majorité visible ». Selon ces prévisions, en 2017 , près de $75 \%$ des Canadiens de minorités visibles devaient vivre dans les trois grands centres urbains du pays: Toronto $(45 \%)$, Vancouver $(18 \%)$ et Montréal $(11 \%)^{8}$.

Si l'art ethnoculturel - en tant que descripteur ou catégorie idéologique de l'expression créative de membres de communautés minoritaires ou, dans une perspective élargie, de non-Européens - est perçu sous un jour négatif au Canada, il serait approprié de s'interroger sur la diffusion, dans les secteurs culturel et éducatif, du savoir relatif à la production culturelle d'artistes non occidentaux ou issus des minorités, dans le cadre du discours ethnonational, sinon ethnonationaliste, dominant. Il conviendrait aussi d'aborder cet enjeu à la lumière du portrait ethnoculturel canadien. En effet, comme l'avance la sociologue Rhoda HowardHassmann, « il existe bel et bien une identité ethnique canadienne $»^{9}$. Plus concrètement, cela soulève plusieurs questions. Quelle matière devrait couvrir un cours sur les histoires de l'art ethnoculturel? Comment sera-t-elle traitée?
5 "Québec: les Noirs et les Arabes forment les deux principales minorités visibles. "STATISTIQUE CANADA.

Recensement de 2006, résultats: La mosaïque ethnoculturelle du Canada.

6 PICHÉ Victor, "Immigration, Diversity and Ethnic Relations in Quebec ", Canadian Ethnic Studies, vol. 34, n 3, 2002, p. 16.
7 STATISTIQUE CANADA. Étude: Population des groupes de minorités visibles au Canada.

8 Ibid.

9 HOWARD-HASSMANN Rhoda E., " "Canadian" as an Ethnic Category: Implications for Multiculturalism and National Unity " Canadian Public Policy, vol. 25, n 4, 1999, p. 523-537. 


\section{Les avantages liés à l'étude des histoires de l'art ethnoculturel}

Qu'entend-on exactement par " histoires de l'art ethnoculturel " ? Quel est l'objet de cette discipline? Selon l'Annuaire des études de $1^{\text {er }}$ cycle de l'Université Concordia, le cours Issues in Ethnocultural Art Histories (" Enjeux des histoires de l'art ethnoculturel ") aborde "les questions d'appartenance ethnique et d'identité culturelle dans les domaines de l'art et de l'histoire de l'art ". Il figure dans le bloc Theory and Criticism ("Théorie et critique"), tout comme son pendant: Postcolonial Theory in Art History ("Théorie postcoloniale en histoire de l'art "). Dans les années 1990, une décennie après l'émergence de politiques identitaires, Concordia a créé les deux cours précités, de même que Topics in Amerindian and Inuit Art (" Aspects des arts inuit et amérindien "). Fait révélateur, ce cours fait partie du bloc Art in Canada ("L'art au Canada »), subsumant en quelque sorte l'ethnicité sous le nationalisme. Pour les départements d'histoire de l'art d'universités européennes et nord-américaines, l'ajout de tels cours s'inscrivait dans une volonté de reconnaître la production artistique jusque-là marginalisée des membres de communautés racialisées. Par ailleurs, il visait à répondre aux exigences du milieu de l'art contemporain ainsi qu'aux pressions mondialistes.

Au milieu des années 1980, le département d'histoire de l'art de l'Université Concordia a réaménagé son programme d'études de premier cycle. Ainsi, il a supprimé la plupart des cours d'introduction spécialisés. De même, il a revu les titres des cours portant sur l'histoire de l'art appelé « extraoccidental ». Il ne subsiste aujourd'hui qu'un anachronisme: le cours Studies in Near Eastern Art and Architecture ("Étude de l'art et de l'architecture du ProcheOrient "), qui se trouve dans le bloc Period Studies ("Études de périodes»). Il s'agit probablement des derniers vestiges d'un certain type de cours qui portaient sur l'histoire de l'art proche-oriental, asiatique ou africain, par exemple. Sinon, aucun titre de cours ne fait maintenant référence à une région hors de l'Europe. Au nombre de ses observations sur les récits et canons en histoire de l'art, Charlotte Bydler souligne qu' « en Occident, les cours universitaires traitant d'histoire de l'art extraoccidental se concentrent généralement sur les arts et cultures des pays d'Asie, d'Amérique latine et d'Afrique, et ce, avant qu'ils soient colonisés par les Européens, qu'ils se modernisent et s'industrialisent ${ }^{10}$ \%. Bien sûr, les pratiques didactiques et institutionnelles actuelles en histoire de l'art remettent souvent en question ce paradigme. Néanmoins, en Occident, les cours d'histoire de l'art extraoccidental sont typiquement offerts dans le cadre de curriculums de l'histoire de l'art qui, depuis leur création, privilégient l'étude de l'art européen. À cet égard, les universités canadiennes, y compris Concordia, ne font pas exception.

D'aucuns estiment que cette évolution graduelle des programmes d'histoire de l'art prouve qu'« au cours des vingt dernières années, les universités canadiennes ont quelque peu atténué leur eurocentrisme traditionnel ${ }^{11}$ ". Cependant, au Canada, la plupart des programmes d'histoire de l'art restent fortement axés sur l'enseignement des canons occidentaux. D'ailleurs, Concordia est au moment d'écrire ces lignes la seule et unique université à proposer un cours dont le titre comporte l'expression ethnocultural art histories (" histoires de l'art ethnoculturel "). J'apporte toutefois une nuance: dans l'ensemble des curriculums, un petit nombre de cours, rarement offerts, se classent sous ce thème même s'ils portent des titres peu évocateurs. Ainsi, des cours traitent de productions liées à l'art diasporique; d'art et de théorie critique de la race; d'arts ethnique et touristique; et moins souvent et moins explicitement - d'art des Premières Nations. Un paradoxe surgit ici: comment l'inclusion généralement fluctuante et sporadique de soi-disant cours sur la " théorie critique de la race " peut-elle aller de pair avec la suppression progressive de programmes d'études spécialisés en art extraoccidental et 
- fait significatif mais certes bizarre - leur réintroduction ultérieure? Par exemple, des activités de recrutement stratégique visent des spécialistes de la modernité, car ces érudits sont jugés aptes à expliquer les contextes non européens et, oserais-je l'écrire?, le concept d'" art mondial ». Cette évolution graduelle et simultanée des programmes d'études soulève la question suivante: quelle est la nature du rapport entre la logique circulaire propre aux approches employées en histoire de l'art mondial et les politiques néolibérales d'inclusion qu'exemplifient censément les cours d'histoire de l'art à portée « ethnoculturelle»?

Je ne me suis pas fixée pour objectif de miner la capacité que possède le cours d'histoire de l'art ethnoculturel à effectuer des analyses artistiques soucieuses des enjeux liés aux identités ethnoculturelles et donc plus intégrantes que celles qui sont menées dans le cadre d'un cours obnubilé par l'étude de la culture et de l'art extraoccidentaux. Par sa structure même, le premier répond plus précisément au besoin incessant de politiques d'inclusion, de reconnaissance et de revendication qui se manifeste dans le domaine des arts. Au contraire, j'entends me montrer constamment attentive à l'éventualité qu'un tel cours, ou un autre comparable, s'accompagne d'une évaluation pédagogique teintée d'ambivalence, de simplisme et de récupération sur de nombreux aspects d'ordre culturel, sociopolitique, économique ou didactique. Le problème transcende les luttes intestines entourant la négociation des diverses modalités de l'engagement culturel. Il réside plutôt dans le fait que ce type de cours se profile, la plupart du temps, comme un dérivé médiocre d'une offre pédagogique plus courante: le cours d'histoire de l'art extraoccidental. D'ailleurs, celui-ci semble avoir été remplacé, notamment à Concordia, par le cours d'histoire de l'art ethnoculturel, même si les sujets qu'abordent l'un et l'autre diffèrent théoriquement.

Comme on le constate souvent dans les répertoires de programmes universitaires, la description du cours offert à Concordia est rédigée dans une optique d'ouverture et d'adaptabilité. Ainsi, la formation pourra intégrer une vaste gamme de sujets et les aborder conformément à la désignation officielle du cours. En outre, la matière variera selon le nombre d'inscriptions, la disponibilité des formateurs et les centres d'intérêt des chercheurs. Dans le cas présent, le texte ne précise pas les ethnicités à l'étude ni leur situation géographique. D'une manière ou d'une autre, l'art et ses perspectives historiques sont liés, sur le plan idéologique, à la formation de l'identité culturelle. Dès lors, le cours se voit doté d'une spécialisation thématique allant du "primitivisme dans l'art occidental " à l'" art islamique du $7^{\mathrm{e}}$ au $15^{\mathrm{e}}$ siècle " ou encore de la "représentation du "sauvage" dans l'art européen " au triptyque " race, citoyenneté et art au Canada ". Ce dernier sujet, qui couvre grosso modo la période entre 1867 et aujourd'hui, constitue du reste le fondement d'un cours que j'ai donné cinq fois depuis 2007: Race, Citizenship and Art in Canada, dans lequel mes étudiants et moi examinions les politiques de représentation, de réparation et de reconnaissance dans les arts dits québécois et canadien. Nous observions plus étroitement l'engagement " canadien " en matière d'art, par l'intermédiaire d'un " groupe des sept " pour le moins différent. En effet, plutôt que de perpétuer la "véritable » image de marque nationale véhiculée par le Groupe des sept, le cours s'attardait à une série de dates clés de l'histoire des politiques d'immigration au Canada et de l'héritage colonial des défricheurs, à des événements qui ont profondément influencé l'énonciation du discours ethnonational, voire ethnonationaliste, sur la « terre de nos aïeux ".

Les 10 dernières années ont été ponctuées de nombreux anniversaires marquants, notamment les 20 ans, en 2008, de la Loi sur le multiculturalisme canadien. De même, l'année 2007 a coïncidé avec le bicentenaire de l'abolition du commerce des esclaves au sein de l'Empire britannique, le centenaire des émeutes anti-Asiatiques de Vancouver, les 60 ans de la Loi sur la citoyenneté canadienne et, en 2017, les 50 ans de la Loi sur l'immigration du Canada. Complexifiant assurément la compréhension d'un art national, ces moments forts fixent le cadre historique du cours Race, Citizenship and Art in Canada. Ainsi, ils balisent 
l'étude de la pratique d'artistes contemporains d'ascendance non occidentale, notamment asiatique et africaine, maintenant établis au Canada. Sur le plan du contenu du cours, les sujets abordés s'étendent des œuvres d'Emily Carr illustrant des mâts totémiques autochtones et de son approche picturale dans la représentation d'immigrants chinois et d'autochtones en Colombie-Britannique, à l'exposition Africville tenue à Halifax ou à l'essai controversé Nègres blancs d'Amérique du journaliste et écrivain québécois Pierre Vallières, en passant par les portraits de femmes noires ou sud-asiatiques qu'a exécutés Joanne Tod. Autrement dit, bien que le cours puisse figurer dans le bloc " ethnoculturel ", son contenu englobe à mon sens les histoires de l'art global.

\section{Les histoires de l'art global au Canada}

Au cœur même de la création du cours, un espace supposément postcolonial appelé "Canada " permet d'élargir le contexte de la présente analyse. Y coexistent des champs d'études et de création artistique; de toute évidence, certains semblent plus pérennes que d'autres. Peu importe, ces champs explorent les complexités et les contradictions que présentent les histoires de l'art autochtone, de l'art diasporique et de l'art extraoccidental. En fait, ces activités s'inscrivent dans une démarche des plus ambitieuses : la " mondialisation de l'histoire de l'art occidental ». Ce processus débute par la " racialisation de l'histoire de l'art ", à la lumière des systèmes de connaissance dominants et de la suprématie blanche de l'art euroaméricain ${ }^{12}$. Comment exactement l'étude de l'art mondial ou de l'art global s'incarne-t-elle actuellement dans les cursus des universités du pays? D'entrée de jeu, je précise que le milieu universitaire canadien ne fournit pour ainsi dire nulle indication quant à une éventuelle reconnaissance de l'histoire de l'art mondial en tant que discipline, peu importe sa dénomination par une expression corollaire comme
" histoires de l'art global ». Au moment de la préparation de ce texte pour sa publication initiale en 2014, aucun programme d'études ainsi nommé n'existait officiellement, et aucun colloque d'importance ne s'était encore penché sur la question au Canada. Cependant, un certain nombre d'érudits, d'étudiants des cycles supérieurs, de membres d'unités d'enseignement interdisciplinaires et de participants à des propositions curriculaires distinctes recourent à cette approche à des fins de recherche. Par conséquent, pour évaluer le mode d'intégration du savoir sur l'art mondial et l'art global dans les cursus qu'offrent facultés et départements de beaux-arts et d'histoire de l'art des universités canadiennes, il faut d'abord et avant tout passer en revue les titres de cours de premier cycle en histoire de l'art. En effet, ces désignations reflètent jusqu'à un certain point une sensibilité pédagogique aux enjeux que posent tant les perspectives postcoloniales que la mondialisation des arts. Une analyse axée sur l'offre de cours depuis 2001 - année de la Déclaration universelle de l'UNESCO sur la diversité culturelle - établirait un bon point de référence.

Des quelque 90 universités canadiennes, environ le quart propose, au premier cycle ou à un cycle supérieur, des programmes menant à un diplôme en histoire de l'art. Les cours dotés d'un titre pertinent sont généralement groupés en six grandes catégories: l'art extraoccidental; les perspectives postcoloniales sur l'art; l'art ethnique, l'art diasporique ou l'art émanant de membres de communautés minoritaires ou racialisées; l'art autochtone ou l'art des Premières Nations; l'art régional ou national (ces cours traitent vraisemblablement de l'identité par rapport au lieu et des enjeux sousjacents); et l'art d'un point de vue international ou global.

Théoriquement, bon nombre de cours à l'appellation conforme, y compris ceux qui abordent les histoires de l'art ethnoculturel, pourraient figurer sous une ou plusieurs 
rubriques. Toutefois, l'énumération succincte de titres de cours d'histoire de l'art offre peu d'information sur le transfert actuel des connaissances thématiques par l'intermédiaire de programmes d'études ("cachés " ou non) d'autres départements d'enseignement, par le truchement de centres et d'activités de recherche facultaires ou universitaires, ou encore au moyen de curriculums variés: études ethniques, études littéraires, écriture migrante, communication et études des médias, études interdisciplinaires, etc. Cela ne signifie pas pour autant que les cours dotés d'un contenu plus traditionnel ne procèdent pas à des comparaisons transculturelles. Après tout, la notion d'art occidental repose sur l'existence de l'art dans le reste du monde. Mais c'est inévitable: même le meilleur programme d'études transculturelles inclura une part de politique purement symbolique. Faisant écho à une opinion partagée par plusieurs spécialistes de la pédagogie, l'historienne de l'art Joanne Sowell affirme que: « L'adjonction d'un contenu multiculturel ne constitue pas une réponse adéquate à la diversité croissante de la population; la pédagogie doit également adopter un caractère multiculture ${ }^{13}{ }^{13}$. Une liste de contrôle ne saurait tenir compte de la possibilité qu'un manque de ressources aggrave des lacunes liées à l'expertise, ni juger du bien-fondé de l'élaboration d'un programme d'études sur la base de préoccupations en matière de racialisation. Nul doute, la discipline nécessite qu'on l'approfondisse de toute urgence, grâce à la mise en œuvre de méthodologies plurithématiques et à l'ajout de ressources.

Au-delà des politiques de comptabilisation, les catégories sont révélatrices en elles-mêmes.

À preuve, trois suppositions peuvent être formulées d'entrée de jeu. Premièrement, et bien que les curriculums ne soient nullement cohérents d'une université à l'autre, les cours d'études postcoloniales témoignent de l'évolution fondamentale de la discipline et de sa volonté de composer avec la crise de représentation culturelle d'une société multiculturelle et multiethnique où les acceptions des nationa- lismes et des identités sont de plus en plus axées sur les collectivités locales. Les cours d'art national basiques reflètent les tensions permanentes entre la préoccupation bien ancrée de définir les concepts nettement controversés d'art et d'histoire de l'art " canadiens " et l'expression d'une diversité culturelle et régionale. Étant donné la suprématie historique de l'Empire britannique, de même que la proximité et la domination du milieu universitaire étasunien dans les dernières décennies, les frontières disciplinaires de l'histoire de l'art canadien doivent perpétuellement être redéfinies.

Deuxièmement, une perception implicite influence l'offre de cours d'histoires de l'art ethnoculturel dans l'université canadienne prétendument postcoloniale. Contrairement à son homologue américaine, celle-ci intègre déjà des curriculums fondés sur la notion de Critical pedagogy of race en culture visuelle et artistique, dans une perspective à la fois analytique et historique. En outre, ces programmes d'études s'appuient sur une histoire de l'art qui fait sienne la théorie critique de la race. Cette spécialité émergente traite d'authenticité, de diversité et de multiculturalisme ${ }^{14}$. Concrètement, l'hypothèse permet d'anticiper le questionnement sur les approches complexes que privilégient les institutions québécoises et canadiennes pour aborder, et parallèlement conserver, les hiérarchies de pouvoir à l'interne, lesquelles reposent sur des facteurs d'ordre culturel, linguistique et racial au sein de la discipline de l'histoire de l'art et, par extension, des programmes d'études qui s'y rattachent. Par exemple, les quelques titres de cours qui incluent le mot " ethnicité ", " race ", « diaspora " ou, tout particulièrement, " ethnoculturel " peuvent laisser entendre deux choses: soit ils reconnaissent le maintien de facto des hiérarchies, soit ils renvoient à la politisation réticente d'une matière « intellectualisée " se fourvoyant dans les affaires temporelles. Par contre, ces titres de cours réfutent tout rejet du fardeau que constitue un déterminisme ethnique incarné par des principes multiculturalistes; 
les différences ethnoculturelles sont considérées ici comme autant de justifications d'une célébration. Des plus réductrices, cette forme d'ethnoculturalisme scinde en différents groupes ethniques les arts, les artistes et les histoires de l'art. Valorisant les liens ethniques, elle empêche leur pleine participation à la construction d'une nation - dans le cas présent, par l'intermédiaire de l'art canadien.

Troisièmement, même si les érudits ne s'entendent pas sur le sens des mots " canadien " (pas plus d'ailleurs qu'» américain " ou " québécois ", dixit Elke Winter) et, de façon plus controversée, " postcolonial ", ces deux notions - en tant que termes favorisant le questionnement - sont encore plus sujettes à caution dans le cas du Québec, car la critique postcoloniale commence à peine à y être pratiquée ${ }^{15}$. Les enjeux liés à la contestation du territoire national se répercutent dans les établissements d'enseignement québécois, outils essentiels à la construction de la nation. Tant sur le plan du contenu que de l'épistémologie, les histoires de l'art ethnoculturel forment par contre un terreau idéal pour l'élaboration d'un discours postethnique et postnationaliste. Étant donné l'impact d'une diversification ethnoculturelle croissante, elles n'échappent toutefois pas à la mainmise du discours ethnonational ou ethnonationaliste.

\section{Portrait du tournant ethnique}

Au nombre des conflits et des crises ethniques récents à la fin des années 2000, un événement des plus instructifs, sinon déchirant, s'inscrit parfaitement dans mon propos: le débat enflammé, hautement médiatisé, sur les questions d'" accommodements raisonnables " et de citoyenneté québécoise qui a éclaté à l'automne 2007. Je donnais pour la première fois le cours Race, Citizenship and Art in Canada quand, au milieu du trimestre, les discussions sur les accommodements raisonnables ont commencé, puis se sont étendues à l'ensemble de la province. Le débat s'était intensifié à la suite des rencontres publiques organisées, durant huit semaines, par la Commission de consultation sur les pratiques d'accommodement reliées aux différences culturelles. Créée par l'État, la Commission était dirigée par Gérard Bouchard, historien et sociologue indépendantiste, et par Charles Taylor, célèbre philosophe d'allégeance fédéraliste ${ }^{16}$. Les coprésidents avaient pour mandat de consulter la population québécoise sur la conduite à privilégier dans les relations avec les minorités ethniques et religieuses. Parce que sa plateforme permettait aussi à des extrémistes défavorables à l'endroit des groupes minoritaires de s'exprimer ${ }^{17}$, la Commission s'était vu accuser, par des associations antiracistes issues d'organismes communautaires et de groupes de promotion de la justice sociale, d'attiser les flammes du racisme, du sexisme et de l'islamophobie. Dans la foulée des consultations, le Parti québécois déposait à l'Assemblée nationale le projet de loi no 195 sur «l'identité québécoise ". Cette proposition lançait l'idée d'une citoyenneté québécoise définie selon le concept de l'ethnicité. Un mois plus tard, l'État soumettait une "nouvelle " constitution québécoise, qui assurerait notamment la protection et la promotion de la culture québécoise et de la langue française. Le projet de loi sur l'identité québécoise visait à faciliter l'élaboration subséquente d'une constitution, où la notion de citoyenneté québécoise reposerait sur des critères linguistiques - la langue française servant de marqueur par excellence de la différence

législation sur la non-discrimination, ce concept s'applique plus particulièrement à la Charte des droits et libertés de la personne du Québec. L'ouvrage Les accommodements raisonnables, publié sous la direction de M. Jézéquel (2007), examine cette question telle qu'elle se posait avant la tenue de la commission Bouchard-Taylor. 17 HEINRICH, J. " Few Racial Slurs at Quebec Accommodation Hearings ", CanWest News Service, 20 décembre 2007. constructing the Canadian multicultural nation ", Nations and Nationalism, 2007, p. 481-503; DESROCHES Vincent, « En quoi la littérature québécoise est-elle postcoloniale?", Quebec Studies, vol. 35, 2003, p. 3.

16 Expression utilisée au Canada, « accommodements raisonnables " renvoie à une théorie voulant qu'en vertu des droits à l'égalité définis à l'article 15 de la Charte canadienne des droits et libertés, des compromis doivent être consentis à diverses minorités ethniques. Dans le contexte de la 
ethnique. La légitimité de la reconnaissance de la culture franco-québécoise était manifeste. Que faut-il retenir de ces événements? Ceux-ci illustrent les moyens qu'a pris la rhétorique civique pour obliger l'opinion publique à trancher entre la protection de la culture québécoise et la sauvegarde des droits des groupes minoritaires, notamment ceux des immigrants. Par ailleurs, ils soulignent à quel point les droits des peuples autochtones sont exclus de l'éternel débat sur le biculturalisme qui opposent la majorité francophone au Québec et le Canada anglais d'un océan à l'autre. Présenté en mai 2008, le rapport final de la Commission concluait " qu'aucune donnée ne permet d'affirmer que la discrimination serait plus présente au Québec qu'ailleurs ". Sur la question des accommodements raisonnables, il préconisait que " tous les Québécois, qu'ils soient de souche ou issus de l'immigration ", fassent preuve de bon sens et vivent en harmonie $^{18}$. Selon la quatrième des 37 recommandations formulées dans le rapport, l'État québécois devait continuer de soutenir l'interculturalisme. Il s'agissait à cette fin de mettre l'accent sur l'intégration au moyen de la francisation, dans un esprit d'égalité et de réciprocité inspiré par la négociation et le compromis, puis d'intégrer ce principe dans une politique officielle.

En 1990, le ministère des Communautés culturelles et de l'Immigration, alors sous la direction du Parti libéral du Québec, publiait un livre blanc. Il y proposait un " contrat moral ", dont le troisième principe prônait d'ériger l'interculturalisme en modèle. L'entente suggérée reconnaissait le caractère pluraliste de la société québécoise "dans les limites qu'imposent le respect des valeurs démocratiques fondamentales et la nécessité de l'échange intercommunautaire $^{19}$ ». Depuis, la position gouvernementale en matière de diversité
18 "The Bottom Line: Use Common Sense", Montreal Gazette, 23 mai 2008.

19 MINISTÈRE DES COMMUNAUTÉS CULTURELLES ET DE L'IMMIGRATION DU QUÉBEC. $A u$ Québec pour bâtir ensemble. Énoncé de politique en matière d'immigration et d'intégration [http://www.midi.gouv.qc.ca/ publications/fr/ministere/Enonce-politique-immigrationintegration-Quebec1991.pdf] reflète une volonté de lier les minorités ethnoculturelles au moyen d'un " contrat moral ". Régi par les valeurs démocratiques libérales fondamentales qui définissent la culture publique commune au Québec, ce serment d'allégeance civique et politique s'étofferait et s'enrichirait, au fil du temps, des particularités des communautés culturelles. Il faut se rappeler que l'État, jusqu'à la fin des années 1990, utilisait le terme " communautés culturelles " pour désigner les groupes dont les membres n'étaient d'ascendance ni française ni anglaise. À l'époque, il s'efforçait de " désethniciser " son approche dans le développement d'un sentiment national au Québec - démarche critiquée parce qu'elle restait strictement axée sur les Québécois de souche, c'est-à-dire ceux qui s'identifiaient comme non immigrants, non autochtones et non anglophones. En d'autres termes, l'État faisait valoir que les Québécois étaient tous liés par une citoyenneté commune, peu importe leurs origines sociales, ethniques ou religieuses, voire leur enracinement historique - autrement dit, leurs " racines ». En conséquence, les références aux communautés ethnoculturelles ont évolué uniquement sur le plan des catégories sociales et administratives, afin d'" intégrer leur différence dans l'élaboration même de l'idée de citoyenneté au Québec ${ }^{20}$ ". Il s'agit certes là d'un modèle dualiste qui, d'une part, inclut dans le débat les Québécois de souche et les communautés culturelles, mais qui, d'autre part, en exclut les Premières Nations, notamment les 11 que reconnaissait le projet de constitution québécoise. En outre, le modèle interculturel est mis en contraste avec le multiculturalisme canadien. En vertu de cette approche, chaque communauté culturelle conserve plus ou moins son mode de vie autonome, mais se conforme à un cadre juridique commun.
20 SALÉE Daniel, "The Quebec State and the Management of Ethnocultural Diversity: Perspectives on an Ambiguous Record ", Belonging? Diversity, Recognition and Shared Citizenship in Canada, 2007, p. 109. 


\section{Fast Forward}

Dans le cas de l'interculturalisme québécois, l'engagement pour l'égalité des chances n'est pas remis en cause. Par contre, comme le souligne Marie McAndrew :

La reconnaissance politique de la réalité des inégalités ethniques et du racisme en est encore à ses premiers balbutiements. Pour tout dire, le débat public n'a même pas commencé. Par ailleurs, on constate une tendance très marquée à transformer les questions socioéconomiques en enjeux culturels. À preuve, l'évolution du concept de citoyennetée ${ }^{21}$.

De fait, pour continuer de citer Mme McAndrew, le «piratage du programme québécois par des personnes préoccupées de langue et de culture a laissé fort peu d'espace à la tenue d'une discussion publique sur les inégalités ${ }^{22}$ ". Si, au Québec, le pluralisme normatif fait grand cas de la diversité ethnique et linguistique, il n'en demeure pas moins que, dans le domaine des arts en général et celui des arts visuels en particulier, la proportion de membres de minorités ethniques ou visibles paraît négligeable. Ainsi, il est moins probable que, comparativement à l'ensemble des Québécois, une personne appartenant à une minorité visible possède un diplôme dans une discipline artistique. L'écart est particulièrement important chez les jeunes ${ }^{23}$. Sur le plan des pratiques culturelles en milieu institutionnel, il existe un fort décalage entre les motivations des autorités gouvernementales et les politiques en vigueur. D'une part, l'État s'est efforcé d'accroître la sensibilisation du public à la diversité ethnoculturelle constituante du Québec dans le monde des arts. D’autre part, la politique culturelle du Québec semble

21 McANDREW Marie, Quebec's Interculturalism Policy: An Alternative Vision. Commentary ", Belonging? Diversity, Recognition and Shared Citizenship in Canada, 2007, p. 147. 22 Ibid.

23 PICHÉ Victor, Op. cit., p. 15; JEDWAB Jack, "Arts and Diversity in Montreal: Preliminary Findings and Recommendations for Future Research. " Document présenté à La Diversité Artistique de Montréal: une Richesse a Partager, Journée de Concertations sur la Diversité Culturelle dans les Arts, Le Conseil des Arts de Montréal, 29 mars 2004. considérer que les artistes - peintres, écrivains, poètes, comédiens... - d'ici sont exclusivement franco-québécois ${ }^{24}$. Cette conception d'une majorité culturellement homogène fait abstraction d'une diversité caractérisée par les minorités, au profit d'une identité nationale idéalisée.

Que cet a priori soit encore en vigueur était bien évident en octobre 2017, alors que plusieurs organismes desservant ou représentant les communautés minoritaires québécoises, réunis sous la bannière du Réseau des artistes pour l'équité, dénonçaient une discrimination systémique de la part du Conseil des arts et des lettres du Québec (CALQ). Malgré le plan d'action pour la diversité culturelle mis en place au CALQ en 2016, certains membres du Réseau, qu'on pense notamment au Festival Accès Asie, déplorent le fait d'avoir complètement perdu leur subvention cet automne-là ${ }^{25}$. Le même mois, le gouvernement du Québec annulait sa consultation publique controversée sur la discrimination systémique et le racisme et la remplaçait par des sessions à huis clos et un forum public. Cette annulation survenait un jour avant que la loi 62, qui demande aux résidents prodiguant et recevant des services publics de le faire avec le visage découvert, soit passée le 18 octobre 2017 avec effet immédiat.

Déjà, 10 ans plus tôt, le cours sur le thème Race, Citizenship and Art (offert dans le contexte conjoint du débat sur les accommodements raisonnables et des vives discussions sur le multiculturalisme et l'interculturalisme) ne pouvait qu'accentuer le fait que les histoires de l'art ethnoculturel offraient de nouvelles possibilités de valoriser l'étude d'œuvres produites par des membres de minorités ethniques. De plus, un tel enseignement permettait de

24 PIETRANTONIO Linda, "Who Is "We"? an Exploratory Study of the Notion of "The Majority" and Cultural Policy", Canadian Ethnic Studies Journal, 2002, p. 151 ; McANDREW Marie, Op. cit., p. 147.

25 MONTPETIT Caroline, "Le CALQ accusé de discrimination systémique ». Le Devoir, 5 octobre 2017. Des organismes artistiques déplorent le manque d'appui à la diversité culturelle [http://www.ledevoir.com/culture/ actualites-culturelles/509612/racisme-au-calq]. 
combattre l'exclusion traditionnelle des réalisations d'artistes de couleur dans le domaine de l'histoire de l'art. Toutefois, les cours d'histoires de l'art ethnoculturel que l'on propose maintenant - et tout particulièrement dans le contexte québécois - doivent composer avec un effectif étudiant bigarré. En effet, les étudiants appréhendent des notions qu'ils ont intériorisées et qui concernent la perception qu'a la majorité culturelle des enjeux liés à l'accueil de la diversité ethnoculturelle et de l'autochtonie: cette perception influence leur propre vision de l'Autre. Pendant ce temps, les avantages potentiels liés à l'examen des dimensions internationales d'activités régionales exacerbent toujours plus les enjeux en présence dans le débat culturel. 


\begin{abstract}
This article offers a critical perspective on the pedagogical direction of what I call "global art histories" in Canada by addressing the apparent impasse posed by the notion of what is euphemistically called "ethnocultural art" in this country. It examines different interpretations of the latter chiefly through a survey of course titles from art history programs in Canada and a course on the subject that I teach at Concordia University in Montreal. Generally speaking, the term "ethnocultural art" refers to what is more commonly understood as "ethnic minority arts" in the ostensibly more derisive discourses on Canadian multiculturalism and cultural diversity. The addition of the term "culture" emphasizes the voluntary self-definition involved in ethnic identification and makes the distinction with "racial minorities." "Ethnocultural communities," along with the moniker "cultural communities" (or "culturally diverse" communities), however, is still often understood to refer to immigrants (whether recent or long-standing), members of racialized minorities, and even First Nations, Inuit, and Métis peoples. Not surprisingly, courses on ethnocultural art histories tend to concentrate on the cultural production of visible minorities or ethnocultural groups. However, I also see teaching the subject as an opportunity to shift the classification of art according to particular geographic areas to consider a myriad of issues in myriad of issues in the visual field predicated on local senses of belonging shaped by migration histories and "first" contacts. As such, ethnocultural art histories call attention to, but not exclusively, the art of various diasporic becomings inexorably bound to histories of settler colonialism and Indigenous sovereignty. This leads me to reflect on some aspects of Quebec's internal dynamics concerning nationalism and ethnocultural diversity that have affected the course of ethnocultural art histories in the province. I argue that the Eurocentric hegemonic hold of ethno-nationalist discourses on art and art history can be seen with particular clarity in this context. Moreover, I suggest that these discourses have hindered not only the awareness and study of art by so-called culturally diverse communities but also efforts to offer a more global, transnational, and heterogeneous (or chiastic) sense of the histories from which this art emerges. In today's political climate, the project that is art history, now more than ever, needs to address and engage with the reverse parallelism that chiastic perspectives on the historiography of contemporary art entail. My critique is forcefully speculative and meant to bring together different critical vocabularies in the consideration of implications of the global and ethnic turns in art and art history for the understanding of the other. I engage in an aspect less covered in the literature on the global turn in contemporary art, namely the ways in which the mutual and dialectical relation between "cultural identity," better described as a "localized sense of belonging" (Appadurai) and the contingency of place may shape, resist, or undermine the introduction of world or global art historical approaches in specific national institutional sites. I argue a more attentive politics of engagement is required within this pedagogical rapprochement to address how histories not only of so-called non-Western art but also diasporic and Indigenous art are transferred holistically as knowledge, if the objective is to shift
\end{abstract}


understandings of the other by emphasizing points of practice in art history as a field, rather than simply the cultural productions themselves.

I propose the term "global art histories" as a provisional rubric that slants the study of globalism in art history to more explicitly include these kinds of located intercultural negotiations. 